\title{
Memento mori
}

\section{Christina Aus der Au}

Prof. de théologie, membre de la rédaction Ethique

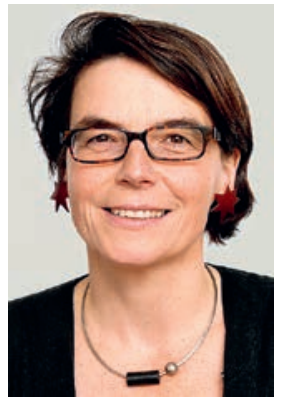

Incroyable. Un collègue que j'appréciais est mort. D'un coup, juste comme ça. Au beau milieu d'une vie bien remplie. Le quotidien s'écroule.

Ce n'est pas la première fois que quelqu'un que je considère comme une relation proche ou même un ami disparaît brutalement. Un avis de décès soudain: infarctus, accident de la circulation, cancer agressif diagnostiqué tardivement ou anévrisme. On appelle ça une mort subite.

Pour la famille, c'est un drame: tout change du jour au lendemain. Et rien ne sera plus jamais comme avant. Savoir que c'est une mort comme nous sommes nombreux à la souhaiter est sans doute une maigre consolation pour les proches. Un décès pendant le sommeil, ou en tout cas rapide. Passer de la santé à l'au-delà sans vraiment s'en apercevoir, de préférence. Tout à fait dans l'esprit de Woody Allen, qui aurait déclaré: «Je ne veux pas dire que j’ai peur de la mort. J'aimerais juste ne pas être là quand elle viendra me trouver."

Vivre sa vie, si possible longtemps, mais sans devenir vieux, sans être victime des tracas de l'âge, ne pas souffrir, ne pas être un fardeau pour sa famille du fait d'une période de maladie difficile entraînant des frais élevés, ne pas se rendre compte du lent vieillissement du corps, du déclin de la vitalité ou, plus inquiétant encore pour beaucoup d'entre nous, de la mémoire, du langage, peut-être même de la réflexion. C'est une éventualité si terrible pour nous qui aimons la vie et les possibilités qu'elle offre que nous pouvons tout à fait trouver un côté positif à une mort subite dans la force de l'âge, du moins en théorie.

Les Grecs le savaient déjà dans l'Antiquité: ceux qui sont aimés des dieux meurent jeunes. Le fardeau de la vieillesse, de la décrépitude, de la dégénérescence leur est épargné. Ils laissent d'eux une image pleine de santé et de joie de vivre. Et c'est à nous, qui sommes restés en arrière, qu'il ne reste plus d'autre choix que de continuer sans eux. «N'oubliez pas: pour notre mort, il suffit de mourir, alors qu'il faut vivre avec celle des autres», a écrit Mascha Kaléko. Et elle sait de quoi elle parle: elle a d'abord perdu son fils unique, puis peu après son mari.

C'est nous, qui sommes restés en arrière, qui tournons et retournons dans nos esprits la dernière conversation, le dernier au revoir, le dernier regard, qui nous torturons avec des "Ah, si seulement j'avais» et des «Ah, si seulement je n'avais pas» et nous efforçons de vivre avec l'impensable. Avec la maigre consolation que c'était une belle mort, une mort rapide pour le défunt, qui lui a sans doute épargné beaucoup de souffrances.

Une lecture récente m'a toutefois amenée à me repencher sur la question. Il s'agit d'une idée moderne, autrefois on priait pour une mort douce et non une mort rapide. «Si tu voulais sans courroux enfin nous ravir à ce monde par une paisible mort», pour reprendre la formule de Matthias Claudius dans son chant du soir La lune s'est levée. Il a écrit ces lignes à une époque où la mort lente ou rapide était terriblement présente, que ce soit du fait de la guerre ou de la maladie. Une mort qui nous arrache à la vie sans préparation, sans possibilité de pénitence et de repentir, sans la promesse du pardon des péchés, est le contraire d'une mort douce dans ce courant de pensée. En paix avec soi, son prochain et, grâce aux derniers sacrements, avec Dieu, on peut en revanche quitter la vie sereinement et en douceur.

Dans notre monde laïque, la réconciliation avec Dieu n'est probablement plus aussi présente. S'exercer à se réconcilier avec ses proches - et peut-être aussi ceux qui le sont moins - est également un aspect d'une bonne vie, telle que l'entendaient les moines du Moyen Age dans le memento mori (souviens-toi que tu vas mourir). Pour les Romains de l'Antiquité aussi, une bonne mort était le point final d'une bonne vie. Le philosophe Sénèque écrit ainsi dans ses lettres à Lucilius: "Avant la vieillesse, je pensais à bien vivre; aujourd'hui, je pense à bien mourir; car c'est bien mourir, que de mourir sans regret.»

Je n'en suis clairement pas encore là. Ce n'était pas davantage le cas de mon collègue. Il avait encore tant de projets. Sa famille avait encore tant besoin de lui. Nous aimerions tellement qu'il soit parmi nous. La mort ne tient pas compte de nos souhaits. Surtout quand elle est rapide.

Je prends donc la résolution, au beau milieu de la vie, de m'exercer de temps en temps à la mort: lâcher prise, me réconcilier, pardonner. Cela ne rendra pas la mort définitive beaucoup plus facile, pas la mienne et encore moins celle des autres. Mais peut-être un peu plus douce, si elle devait être subite. 